\title{
Functional Analysis of a Pomegranate (Punica granatum L.) MYB Transcription Factor Involved in the Regulation of Anthocyanin Biosynthesis
}

\author{
Ghazale Khaksar 1, Badraldin Ebrahim Sayed Tabatabaei 2,3*, Ahmad Arzani ${ }^{1}$, Cyrus Ghobadi ${ }^{4}$, Esmaeil \\ Ebrahimie 5,6
}

1Department of Agronomy and Plant Breeding, College of Agriculture, Isfahan University of Technology, Isfahan, Iran
2Department of Biotechnology, College of Agriculture, Isfahan University of Technology, Isfahan, Iran
${ }^{3}$ Department of Molecular Plant Physiology, Utrecht University, Padualaan 8, 3584CH, Utrecht, The Netherlands
${ }^{4}$ Department of Horticulture, College of Agriculture, Isfahan University of Technology, Isfahan, Iran
${ }^{5}$ Department of Crop Production and Plant Breeding, College of Agriculture, Shiraz University, Shiraz, 73761, Iran
${ }^{6}$ Discipline of Genetics, School of Biological Science, University of Adelaide, Adelaide, 5001, SA, Australia
${ }^{*}$ Corresponding Author: Badraldin Ebrahim Sayed Tabatabaei, Department of Biotechnology, College of Agriculture, Isfahan University of Technology,
Isfahan, Iran. Tel: +98-3133913344, Fax: +98-3133912254, E-mail: sayedt@cc.iut.ac.ir

Received: October 23, 2014; Revised: December 27, 2014; Accepted: February 09, 2015

Background: Pomegranate fruit (Punica granatum L.) is a rich source of anthocyanin pigments resulting in vibrant colours and anti-oxidant contents. Although the intensity and pattern of anthocyanin biosynthesis in fruit are strongly influenced by R2R3-MYB transcription factors, little is known about the regulation and role of MYB in anthocyanin pathway of pomegranate.

Objectives: The present study was conducted to elucidate the relationship between the expression of MYB transcription factor and the anthocyanin accumulation during the colour development phase of pomegranate fruits.

Materials and Methods: In this work, R2R3-MYB transcription factor (PgMYB) was isolated and characterized from pomegranate skin through RACE-PCR. The expression of PgMYB gene was monitored in three distinct pomegranate accessions with distinctive skin colour and pattern by semi-quantitative RT-PCR.

Results: The results indicated a strong association between skin colour in mature pomegranate fruits with the $P g M Y B$ transcripts. The highest expression level of $P g M Y B$ gene was observed in Poost Siyah Yazd (dark purple skin) throughout the ripening process. Furthermore, comparison of PgMYB amino acid sequences with those of R2R3-MYB family in grapevine, eucalyptus, peach, cacao, populus and Arabidopsis demonstrated that this protein shares high similarity (75$85 \%$ amino acid identity) with their conserved MYB domain. Computational structure prediction of PgMYB showed that the three conserved amino acids (Asn, Lys and Lys) are present in the same position of the MYB domain.

Conclusions: It is speculated that $P g M Y B$ gene influences the fruit colour and could be used to improve the accumula-tion of anthocyanin pigments in the pomegranate fruit.

Keywords: Anthocyanin biosynthesis; MYB transcription factor; Pomegranate

\section{Background}

Land plants possess successful mechanisms for responding to environmental challenges. In these elaborate systems, transcriptional regulation plays critical roles due to their broad adaptation and coordinately modulates of hundreds of different genes within regulatory networks. Transcription factors are powerful means for regulation of gene expression and consist of at least four distinct domains, DNA binding domain, nuclear localization signal (NLS), transcription activa- tion domain, and oligomerization site, which operate together to control many physiological processes (1). Based on the specific conserved DNA-binding domains, the transcription factor genes can be classified into different families or super-families (2). The MYB transcription factor superfamily is a group of functionally diverse proteins found in both plants and animals. The distinguishing characterization of this family is possession of one to four or more imperfect MYB repeats (R), which can function synergistically 
or discretely in DNA binding and protein-protein interactions, respectively (3). The MYB domain consist of tandem repeats of about 50 amino acids located near the protein's amino terminus that is the basis of the classification of this protein family. MYB domain consists of three $\alpha$-helices, with the second and third helices forming a helix-turn-helix (HTH) structure to intercalate in the major groove of target DNA (1). Activation domain that is resided at $\mathrm{C}$-terminus varies considerably between MYB proteins. This domain plays wide range of regulatory roles in $M Y B$ gene family $(4,5)$.

MYB proteins have been categorized into three major groups: R2R3-MYB, with two adjacent repeats; R1R2R3-MYB, with three adjacent repeats; and a heterogeneous group collectively referred to as the MYBrelated proteins, which usually but not always contain a single MYB repeat (3-5). In plants, a large number of MYB proteins have essential roles in many significant physiological and biochemical processes, including regulation of primary and secondary metabolisms, control of cell development and cell cycle, and response to various biotic and abiotic stresses $(2,6,7)$. In particular, interacting R2R3-MYB and bHLH type TFs, form a complex with WD40 proteins (termed the MBW complex), to control the anthocyanin pathway genes $(8,9)$. MYB transcription factors that regulate the expression of anthocyanin biosynthetic genes have been identified from many species such as Arabidopsis MYB75 and AtMYB90 (7, 10), petunia AN2 (11), grapevine MYBA1 and MYBA2 (12-14), maize $C 1$ family (15), apple MYB10/MYB1/ MYBA $(16,17)$ and legume LAP1 (18).

As powerful antioxidants, anthocyanins exhibit a wide range of protective effects with potential benefits for human health (19). Anthocyanins, as food colourants and beneficial chemo-protective agents are valuable in producing healthier food $(20,21)$. Anthocyanins, a class of flavonoids with absorbance in the visible range, are also responsible for the red, purple, and blue colours in many fruits, vegetables, cereal grains, and flowers $(22,23)$. These valuable pigments play important roles in the signaling between plants and microorganisms, plant defense mechanisms, auxin transport, UV protection, recruitment of pollinators and seed dispersers (24). Up to date, the biosynthesis of the anthocyanin pigments have been studied in various plant species $(23,24)$. The production of anthocyanin is correlated with the activity of enzymes such as phenylalanine ammonia-lyase (PAL), chalcone synthase (CHS), flavanone 3-hydroxylase (F3H), dihy- droflavonol 4-reductase (DFR), leucoanthocyanidin dioxygenase (LDOX, also called anthocyanidin synthase, ANS) and UDP-Glucose: flavonoid-3-O-galactosyl transferase (UFGT) in various plants.

Pomegranate (Punica granatum) fruits are recognized for their high anthocyanin content accumulation in skin and arils $(23,25)$. In pomegranate fruits, the degree of pigmentation is crucial factor that determine the appeal of fruits to the customers, influence the antioxidant activity and protect cellular compounds against biotic stress and radiation damage $(26,27)$. The different pomegranate accessions exhibit variable patterns of skin colour; from yellow, through pink and red to deep purple (28). One possible reason for the observed colour variation in pomegranate skin can be related to differences in the expression of regulatory genes (23). Analysis of pomegranate $M Y B$ gene showed that the expression level of $P g A n 2$ is correlated with the skin colour (29). In many plants, different $M Y B S$ are involved in transcriptional regulation of anthocyanin production. In maize, two classes of regulatory genes ( $C 1$ and $R$ gene families) control expression of anthocyanin biosynthetic genes $(30,31)$. Also, in grapes, $V v M Y B A 1$ and $V v M Y B A 2$ had a regulatory effect on fruit colour (12-14). Therefore, it seems that plant pigmentation patterns are controlled by more than one MYB protein.

In the present work, homologous of $V v M Y B A 1$ and $V v M Y B A 2$ were identified from several plant species, including eucalyptus (Eucalyptus grandis) peach (Prunus persica), cacao (Theobroma cacao), populus (Populus trichocarpa) and Arabidopsis thaliana. They encode an R2R3 MYB domain protein with high similarity to the grape. An R2R3 MYB regulator was isolated and characterized from pomegranate. The changes in expression of $M Y B$ was compared during fruit development in three cultivars, namely Shirin Shabad Shiraz (pink), Bozi Isfahan (red) and Poost Siyah Yazd (dark purple).

\section{Materials and Methods}

\subsection{Plant Material and Growth Conditions}

Three pomegranate accessions, Poost Siyah Yazd (dark purple skin), Bozi Isfahan (red skin) and Shirin Shabad Shiraz (pink skin) were sampled from the pomegranate tree collection in the Agricultural and Natural Resources Research Center of Isfahan (http://esfahan.areo.ir). Flowers and fruits were collected between May till October. The samples were selected from four different developmental stages: 
flower (stage 1), young fruit (stage 2), nearly mature fruit (stage 3) and ripened fruit (stage 4). In Bozi Isfahn, the skin colour in the developing fruit changes gradually from the prominent orange-red (stage 1) to green (stage 2). At fruit maturation and ripening (stage 3 and 4) the colour changed to red. Post Siyah Yazd acquired its black skin very early and remained black until ripening time. In contrast, the colour of Shirin Shabad Shiraz remained green at stages 2 and 3, but turned pink at fruit ripening stage (stage 4). For each sampling stage (1-4), flowers/fruits were collected from three trees and considered as biological replicate. The fruit skin was removed and stored immediately at $80^{\circ} \mathrm{C}$ for further analysis.

\subsection{Oligonucleotide Design}

The degenerate primers were designed according to the conserved regions in R2R3 domain of MYB family from grapevine, eucalyptus, peach, cacao, populus and Arabidopsis that were used for polymerase chain reaction (PCR). To perform semi-quantitative RT-PCR experiments, specific primers were designed from conserved domains of R2R3 MYB protein (Table 1).

\subsection{Nucleic Acid Isolation}

Total RNA was extracted from Poost Siyah Yazd, Shirin Shabad Shiraz and Bozi Isfahn accessions, from four different developmental stages of flower and fruit skin with the method described for peach trees by (32). Tissues from at least three fruits from three different trees of the same accession and sampling date were combined and extracted. The isolated RNAs were quantified by spectrophotometry (Beckman DU-64 spectrophotometer, USA), the quality was assessed by the absorbance ratios of A260/A280 nm (1.8-2.2) and A260/A230 $\mathrm{nm}(\geq 1.8)$ and by the demonstration of intact ribosomal RNA bands in agarose gel electrophoresis. Each total RNA sample was incubated with 1 U RNase-free DNase I (Fermentas Co.
Germany) for $30 \mathrm{~min}$ at $37^{\circ} \mathrm{C}$ to remove co-extracted genomic DNA.

Genomic DNA was extracted from fresh young leaves using a CTAB-PVP method as described in (33) and quantified by agarose gel electrophoresis and spectrophotometrically.

\subsection{Isolation of PgMYB}

A combination of RT-PCR, $3^{\prime}$ and $5^{\prime}$-RACE techniques were used to isolate the full length of $P g M Y B$, from Poost Siyah Yazd skin tissue. Initially, a $169 \mathrm{bp}$ fragment was amplified based on the degenerate primers (Table 1). First-strand cDNA was prepared from $1.0 \mu \mathrm{g}$ of total RNA using MMLV reverse transcriptase and oligo (dT) according to the manufacturer's instructions (cDNA kit; Thermo scientific). PCR conditions were as follows: $95^{\circ} \mathrm{C}$ for $4 \mathrm{~min}$ followed by 30 cycles of $95^{\circ} \mathrm{C}(30 \mathrm{~s}), 58^{\circ} \mathrm{C}(30 \mathrm{~s})$ and $72^{\circ} \mathrm{C}(60$ s) with a final extension at $72^{\circ} \mathrm{C}$ for $7 \mathrm{~min}$ and $4^{\circ} \mathrm{C}$ for $30 \mathrm{~min}$. DNA fragment is cloned with the InsT/Aclone PCR cloning kit according to the manufacturer's instructions (Thermo Scientific, Germany). Sequence analysis allowed the design of nested primers for 3' and 5'-RACE reactions (5'/3' RACE Kit, Roche Applied Science, Germany). To isolate full length sequence of $P g M Y B$ cDNA.

\subsection{Gene Expression Analysis}

The expression level of MYB regulator was evaluated using semi-quantitative reverse transcriptasepolymerase chain reaction (RT-PCR). Samples were normalized with $18 \mathrm{~S}$ rRNA as a reference gene for constitutive expression. For RT-PCR assays, PCR reactions were performed with $27,30,33$, and 40 cycles to ensure that amplifications were within the linear range. PCR conditions were as follows: $95^{\circ} \mathrm{C}$ $(30 \mathrm{~s}), 58^{\circ} \mathrm{C}(30 \mathrm{~s})$ and $72^{\circ} \mathrm{C}(60 \mathrm{~s})$ with a final extension at $72^{\circ} \mathrm{C}$ for $7 \mathrm{~min}$. Validation of the correct PCR conditions to appropriately determine level of gene

Table 1. Oligonucleotide primers used for RACE-PCR and RT-PCR experiments

\begin{tabular}{lcccc}
\hline Gene & Name & Sequence (5' to 3') & $\begin{array}{c}\text { Annealing } \\
\text { temperature }\left({ }^{\circ} \mathbf{C}\right)\end{array}$ & $\begin{array}{c}\text { Amplicon Size } \\
\text { (bp) }\end{array}$ \\
\hline PgMYB & MYB-1 & GCA GGI CTY AAY AGI TGY AG & 52 & 198 \\
& MYB-2 & ATT CCA RTA RTT YTT RAT YTC & & 123 \\
& 5-MYB & TCT GCA TCT GTT CAG TCC TG & 56 & 301 \\
& 3-MYB & GAC TGA TAA CGA GAT TAA GAA C & 54 & 262 \\
\hline \multirow{3}{*}{ MYMB } & MYB-F & GGT TGC AGG ACT GAA CAG ATG C & 56 & \\
& MYB-R & GCT GGA ACT TCT CTG GAT TTC TC & & \\
\hline
\end{tabular}


expression was performed by making serial dilutions of the cDNA samples and consequently the band intensity was determined on $1 \%(\mathrm{w} / \mathrm{v})$ agarose gels. Densitometric analyses were performed using EZQuant-Gel software. The obtained data were expressed as the mean and standard error (SE) of five PCR replicates.

\subsection{In Silico Characterization of PgMYB}

To provide comprehensive information for MYB family, the BLAST program was used to search homologues in TF database PlantTFDB version 3.0 (34). A multiple sequence alignment of the deduced amino acid sequence of PgMYB and other MYB proteins associated with regulation of the anthocyanin biosynthetic pathway was constructed using Clustal Omega (http://www.ebi.ac.uk/Tools/msa/clustalo/). For pretty printing multiple alignment output, BoxShade program was used (http://www.ch.embnet.org/software/ BOX_form.html).

Primary, secondary and tertiary structure analysis of PgMYB and other MYB proteins were performed to identify the structurally similar protein templates on the ExPASy proteomics server (http://www.expasy.org). The tertiary structure of PgMYB was modeled using the tool PHYRE 2.0 (35); PHYRE offered the homology modelling of PgMYB that shared the high similarity with the animal c-MYB DNA-binding domain. The crystal structure of this protein was previously resolved with its DNA consensus motif (AACNG) by heteronuclear multidimensional NMR (36). The Docking studies were used to check the conformation and binding mode of DNA with PgMYB using Hex 6.3 software.

\section{Results}

\subsection{Isolation and Characterization of PgMYB}

The full-length size of the $P g M Y B$ was found to be $930 \mathrm{bp}$; with $567 \mathrm{bp}$ open reading frame encoding a protein with 189 amino acid residues. Comparison of the deduced PgMYB protein sequence with the R2R3MYB family in eucalyptus, grapevine, peach, cacao, populus and Arabidopsis revealed that this protein shares high sequence similarity $(75-85 \%$ amino acid identity) with their conserved MYB DNA-binding domain (Figure 1). Moreover, multiple sequence alignment data showed a high sequence similarity of PgMYB with grape proteins: VvMYBA1 and VvMYBA2 (Figure 1). Additionally, a highly conserved DNA-binding domain was noted at the N- ter- minus of all the analyzed sequences and for the rest of the sequence of these anthocyanin regulatory proteins the homology was quite high (Figure 1).

Comparison between the genomic DNA and the cDNA sequences (data not shown) indicated that $P g M Y B$ gene contains a single intron within the protein coding region, similar to $V v M Y B A 1$ isolated from grapevine (37), and other PgMYB homologs (Data not shown). $P g M Y B$ sequence was deposited to GenBank database under accession number KF631413.

\subsection{D-Structural Modelling of PgMYB}

The tertiary structure of DNA-PgMYB complex was predicted based on the experimentally solved DNA-animal c-MYB domain with an E-value of 3.8e24 within an estimated precision of $99.9 \%$ (Figure $2 \mathrm{~A}$ ). The two protein sequences were $35 \%$ alike using amino acid sequence alignment. It is intriguing that the animal c-MYB region contains a conserved R2R3MYB domain that exhibits structural similarity to plant R2R3-MYB DNA binding domains. In fact, this NMR showed that ACG nucleotides in the motif (AACNG) served as the core recognition motif, critical for c-MYB binding. In order to identify target DNA-binding sites for PgMYB, the DNA consensus motif (AACNG) of c-MYB was docked into the predicted binding sites of the PgMYB model (Figure 2B). Based on the previous studies, several specific interactions can be predicted between amino acids and base pairs in the region covered by R2R3 (38-40). In particular, these complex structure is characterized for the specific interactions of the three bases at positions 4,6 , and 8 in the core AACTG; (A4+, G6-, and G8+ are interacting with Asn-183, Lys-182, and Lys-128, respectively) (41). Interestingly, the model of PgMYB revealed that these amino acids were extremely conserved in PgMYB domain and consist of Asn-97, Lys96, and Lys-42, respectively. In addition, computational structure prediction of the homologues of PgMYB showed that these conserved amino acids are present in the same position of their MYB domain. In fact, the alignment data (Figure 1) demonstrated that the level of conservation in third repeat (R3) is even greater and involves in DNA binding. Several models assumed that both R2 and R3 contain three helices and that each third helix is the recognition helix located in the major groove of DNA. Based on the tertiary structure of DNAPgMYB complex, three conserved amino acids (Asn, Lys and Lys) involve in DNA binding. Also, these amino acids are conserved in MYB family from eucalyptus, grapevine, peach, cacao, populus and Arabidopsis. 


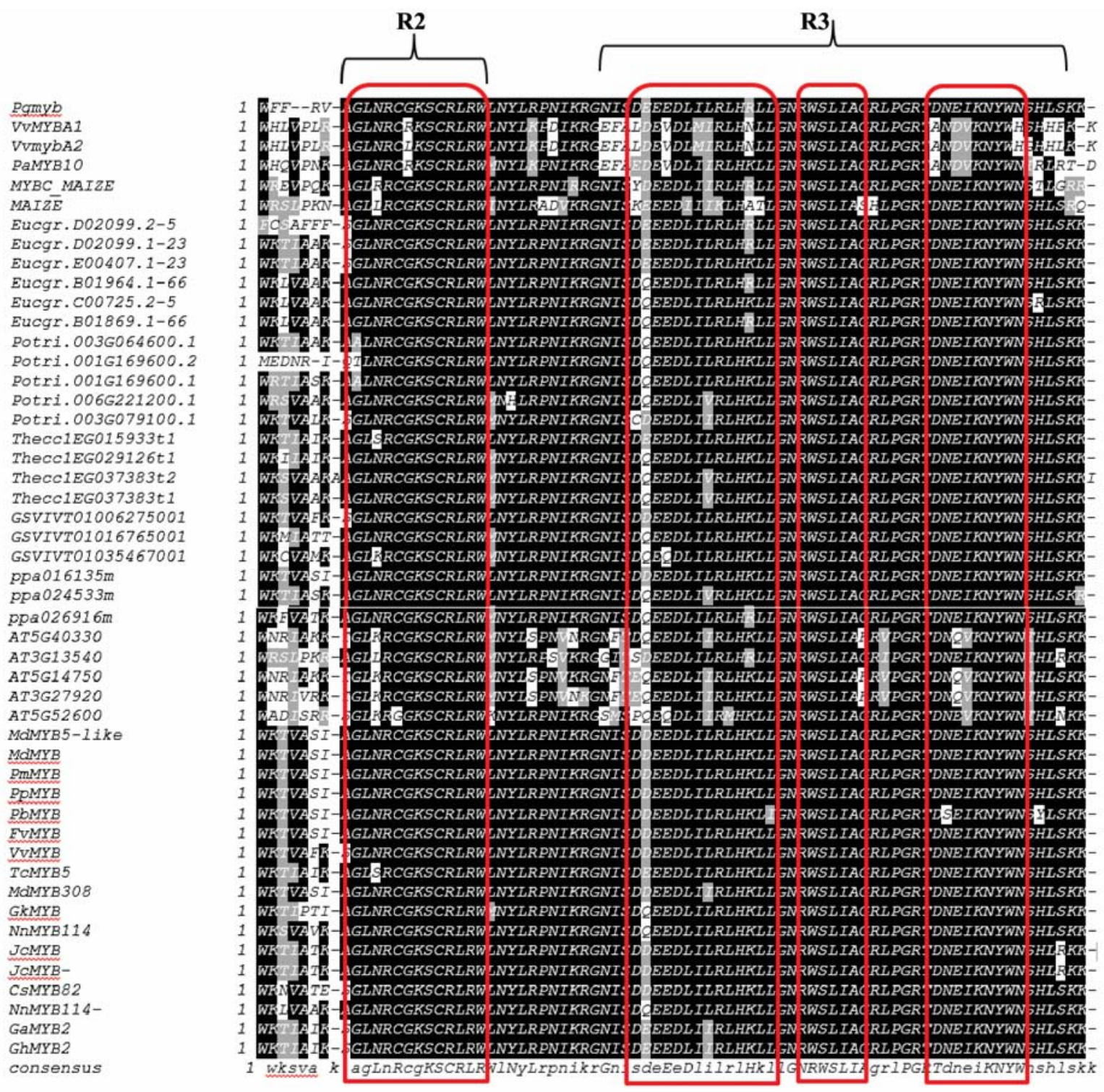

Figure 1. Alignment of deduced amino acid sequences of PgMYB homologous proteins. The R2R3MYB domain are indicated with open boxes. Identical residues are highlighted on a black background, while similar residues and gaps are highlighted on a gray and white background, respectively. The name of the proteins, their source and GenBank accession numbers are as follows: PgMYB isolated from Punica granatum (KF631413); VvMYBA1 from Vitis vinifera (BAD18977); VvMYBA2 from Vitis vinifera (BAD18978); PaMYB from Prunus persica; MYBC MAIZE from Zea mays (P10290); Eucgr.D02099.2-5 from Eucalyptus grandis; Eucgr.D02099.1 from Eucalyptus grandis; Eucgr.E00407.1 from Eucalyptus grandis; Eucgr.B01964.1 from Eucalyptus grandis; Eucgr.C00725.2 from Eucalyptus grandis; Eucgr.B01869.1 from Eucalyptus grandis; Potri.003G064600.1 from Populus trichocarpa; Potri.001G169600 from Populus trichocarpa; Potri.001G169600.1 from Populus trichocarpa; Potri.006G221200.1 from Populus trichocarpa; Potri.003G079100.1 from Populus trichocarpa; Thecc1EG015933t1 from Theobroma cacao; Thecc1EG029126t1 from Theobroma cacao; Thecc1EG037383t2 from Theobroma cacao; Thecc1EG037383t1 from Theobroma cacao; GSVIVT01006275001 from Vitis vinifera; GSVIVT01016765001 from Vitis vinifera; GSVIVT01035467001 from Vitis vinifera; ppa016135m from Prunus persica; ppa024533m from Prunus persica; ppa026916m from Prunus persica; AT5G40330 from Arabidopsis thaliana; AT3G13540 from Arabidopsis thaliana; AT5G14750 from Arabidopsis thaliana; AT3G27920 from Arabidopsis thaliana; AT5G52600 from Arabidopsis thaliana; MdMYB-like5 from Malus domestica (XP 008356551.1); MdMYB from Malus domestica (XP_008364573.1); PmMYB from Prunus mume (XP_008238440.1); PpMYB from Prunus persica (EMJ11205); PbMYB from Pyrus x bretschneideri (XP 009369005); FvMYB from Fragaria vesca (XP 004299414); VvMYB from Vitis vinifera (XP 010646852); TcMYB5 from Theobroma cacao (EOY24284.1); MdMYB30 $\overline{8}$ from Malus

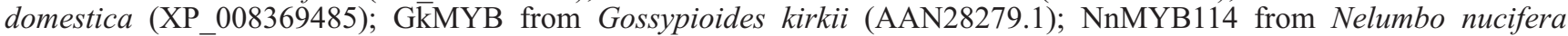

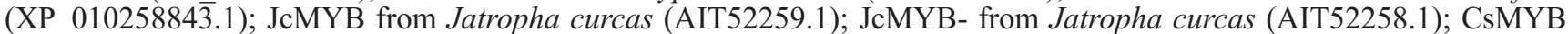
from Citrus sinensis (XP_006477150); NnMYB114- from Nelumbo nucifera (XP_010264377.1); GaMYB from Gossypium arboretum (AAU12248) and GhMYB from Gossypium hirsutum (AFN53779.1) 


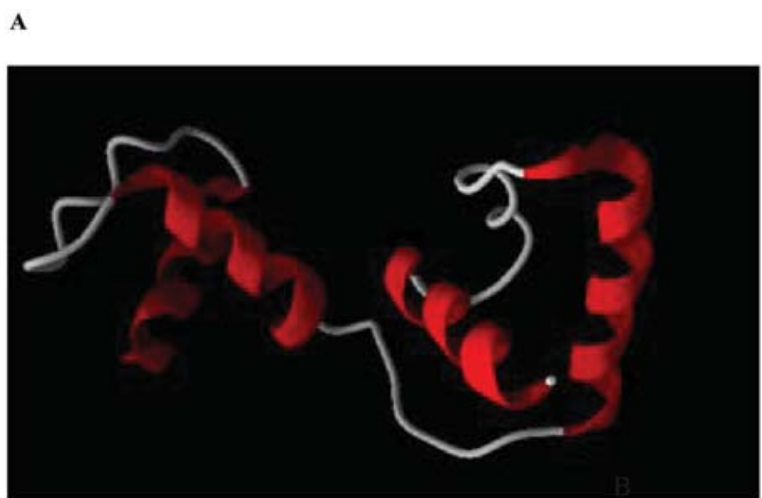

B

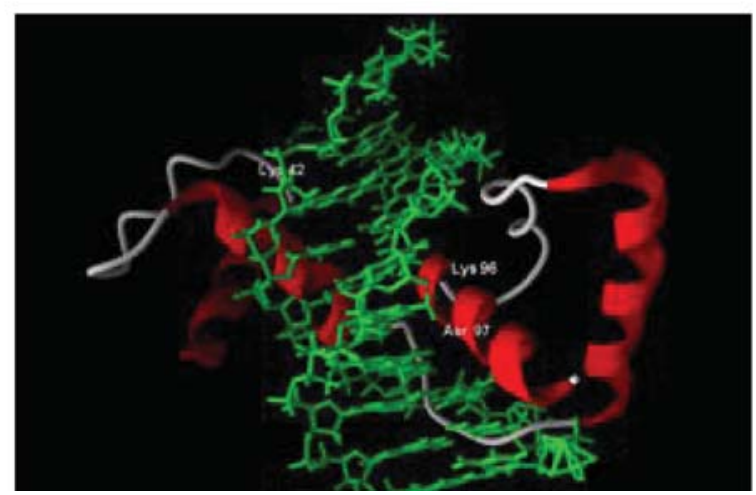

Figure 2. A: The tertiary structure of PgMYB transcription factor, B: The tertiary structure of DNA-PgMYB complex

\subsection{Transcriptional Profile of PgMYB Gene}

The expression patterns of regulatory gene, $P g M Y B$ was monitored in three pomegranate accessions and the results are presented in Figure 3. The expression data showed that $P g M Y B$ transcripts were present at low levels in flowers. In comparison with other accession, Poost Siyah Yazd showed a slight increase of $P g M Y B$ messenger during the early stages of development (stage 2). However, maximum transcript levels were observed in mature fruits (stage 4). At this stage, the skin colour is very dark purple. In controversy, in Shirin Shaabad Shiraz PgMYB transcript was detected at low levels during the fruit development process. In third accession (Bozi Isfahan), PgMYB was expressed at low level in young developing fruits (stages 2), but gradually increased as the fruit matured (stage 3). Expression of this gene reached to its peak when the fruit became over-ripened (stage 4). Comparison the steady state level of PgMYB mRNA in Poost Siyah Yazd with Bozi Isfahan showed that the expression of this gene was remarkably higher in coloured skin of Poost Siyah Yazd.

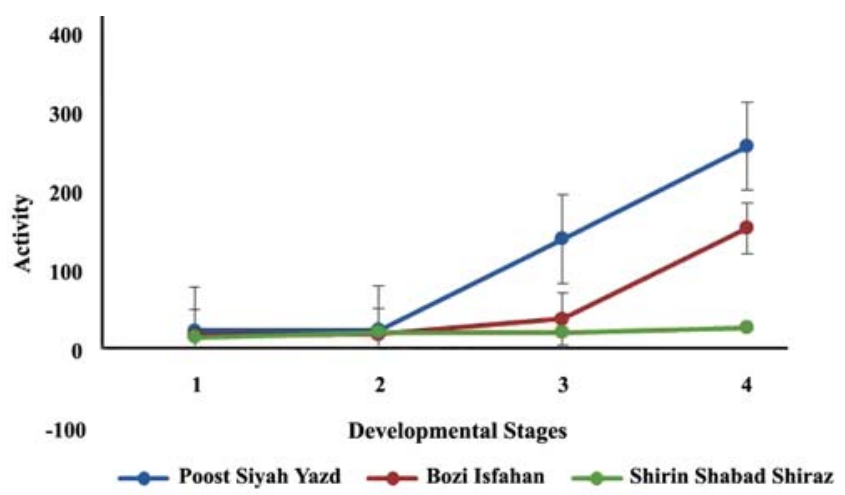

Figure 3. Expression of $P g M Y B$ gene during fruit development in three different pomegranate accessions, Poost Siyah Yazd, Bozi Isfahn and Shirin Shabad Shiraz. Semi-quantitative RT-PCR analysis was performed and samples were normalized with $18 \mathrm{~S}$ rRNA as a reference gene for constitutive expression

\section{Discussion}

MYB/bHLH/WD40 complex is necessary for the appropriate regulation of the anthocyanin biosynthesis various plant species (8). It is known that the difference in the regulatory mechanism mirrors in variation of pigmentation patterns. The present study demonstrated that the highest expression level of $P g M Y B$ gene was observed in the skin of dark purple fruits (Poost Siyah Yazd). Also, its transcript level increased simultaneously with anthocyanins accumulation in this tissue suggesting that $\operatorname{PgMYB}$ is involved in anthocyanins biosynthesis in pomegranate fruits. Indeed, the colour of mature pomegranate fruits reflected the activity of regulatory gene $P g M Y B$ in anthocyanin biosynthesis. Similar results have been obtained in the developmental studies of Arabidopsis (42), maize (15), apple (43), and petunia (11) and grapevine (37). Another point of view, the plant MYB proteins play a crucial role in determining the spatial and temporal patterning of anthocyanin production in most plant species (44). In apple, it was proposed that the MYB component involved in anthocyanins regulation conferred the light responsiveness. In Perilla, MYB factor, MYB-P1, and one of the bHLH factors named MYCF3G1 are required for anthocyanin synthesis under light condition (45). Moreover, the $M Y B$ genes $C l$ and $\mathrm{Pl}$ are light induced in maize (15). Further, some members of the MYB family show tissue-specific and developmental pattern of expression. Here, the expression level of regulatory gene $P g M Y B$ remarkably increased in the skin of pomegranate during fruit 
ripening (Poost Siyah Yazd and Bozi Isfahan). These finding are in agreement with Gil's (1995) reports, which showed skin colour continuously enhanced during the maturation of pomegranate fruits (26). In ripening apple fruit the transcription of $M d M Y B 1$ was correlated with anthocyanin production in red skin sectors of fruit (17). Also, in maize, the $C l$ gene modulates the expression of structural genes for enzymes involved in anthocyanin production during seed development (15). In other plants, evidence of tissue-specific regulation are reported as well. For instance, GhMYB 7/9 are specifically expressed in flowers and fibers, and their expression in fibers is developmentally regulated (1).

The current study demonstrated that PgMYB protein contains the R2R3 imperfect repeats, responsible for binding to target DNA sequences and is highly conserved among R2R3-MYB proteins. It is well documented that gene-specific regulation is controlled by transcription factors that interact with specific short conserved DNA sequences, known as cis-elemnets (46). Herein, the 3D-structural modelling predicted interactions between PgMYB amino acid residues and corresponding nucleotides in the DNA targets (AACNG). PgMYB transcription regulator like other R2R3MYB gene families in plants is characterized by the MYB domain. This domain consists of two imperfect repeats referred to as $\mathrm{R} 2$ and $\mathrm{R} 3$, each forming a helix-turn-helix structure of about 53 amino acids (1). In addition, R2 and R3 motifs intercalate in the major groove, so that these recognition helices interact directly with each other to bind to the specific base sequence, AACNG cooperatively (36). Surprisingly, promoter analysis of structural genes involved in anthocyanin pathway showed that CCAAT-box (CAACGG motif) is present in promoter region of CHS, F3H and UGFT in Arabidopsis, CHS in grapevine and corn. This conserved motif that is known as stress-responsive element (MYBHv1 binding site) is located upstream of regulatory genes such as AtMYB12, AtMYB113 (42) and VvbHLH (47) (data not shown). Based on homology modelling, PgMYB protein bind to this conserved motif, which is present in promoter of structural genes. Therefore, it is expected that PgMYB transcription factor regulate the anthocyanin pathway under developmental stages and/or environmental conditions.

In conclusion, understanding the specific role of MYB to activate pigmentation awaits further experiments. However, it is known that the abundance of anthocyanins in fruits is tightly regulated by three classes of regulatory factors, MYB, bHLH and WD40 proteins. Therefore, PgMYB protein appears to regulate anthocyanins biosynthesis during reproductive stages of pomegranate accessions. The obtained data revealed that PgMYB regulator bind to specific DNA sequences within the promoter regions of anthocyanin biosynthetic genes to enhance their expression. Further, the results showed that the MYB domain is well conserved among plants such as Arabidopsis, grapevine, peach, cacao, populous and pomegranate.

The current findings may also facilitate our ability to develop new pomegranate accessions with enhanced anthocyanin content or improved fruit colours by utilizing molecular-genetic approaches.

\section{References}

1. Du H, Zhang L, Liu L, Tang XF, Yang WJ, Wu YM, et al. Biochemical and molecular characterization of plant MYB transcription factor family. Biochemistry 2009;74(1):1-11. DOI: 10.1134/S0006297909010015

2. Yanhui C, Xiaoyuan Y, Kun H, Meihua L, Jigang L, Zhaofeng G, Zhiqiang L, Yunfei Z, Xiaoxiao W, Xiaoming Q, Yunping S, Li Z, Xiaohui D, Jingchu L, Xing-Wang D, Zhangliang C, Hongya G, Li-Jia Q. The MYB transcription factor superfamily of Arabidopsis: expression analysis and phylogenetic comparison with the rice MYB family. Plant Mol Biol. 2006;60(1):107-124. DOI: 10.1371/journal.pone.0118565.

3. Rosinski JA, Atchley WR. Molecular evolution of the Myb family of transcription factors: evidence for polyphyletic origin. JMol Evol. 1998;46(1):74-83. DOI: 10.1007/PL000062 85.

4. Jin H, Martin C. Multifunctionality and diversity within the plant MYB-gene family. Plant Mol Biol. 1999;41(5):577-585. DOI: 10.1023/A:1006319732410.

5. Stracke R, Werber M, Weisshaar B. The R2R3-MYB gene family in Arabidopsis thaliana. Curr Opin Plant Biol. 2001;4(5):447-456. DOI: 10.1016/S1369-5266(00)00199.

6. Araki S, Ito M, Soyano T, Nishihama R, Machida Y. Mitotic cyclins stimulate the activity of c-Myb-like factors for transactivation of $\mathrm{G} 2 / \mathrm{M}$ phase-specific genes in tobacco. $J$ Biol Chem. 2004;279(31):32979-32988. DOI: 10.1074/jbc. M403 171200.

7. Borevitz JO, Xia Y, Blount J, Dixon RA, Lamb C. Activation tagging identifies a conserved MYB regulator of phenylpropanoid biosynthesis. The Plant Cell. 2000;12(12):23832393.

8. Gonzalez A, Zhao M, Leavitt JM, Lloyd AM. Regulation of the anthocyanin biosynthetic pathway by the TTG1/bHLH/Myb transcriptional complex in Arabidopsis seedlings. Plant J. 2008;53(5):814-827. DOI: 10.1111/j.1365313X.2007.03373.

9. Quattrocchio F, Wing JF, Va K, Mol JNM, Koes R. Analysis of bHLH and MYB domain proteins: species-specific regulatory differences are caused by divergent evolution of target anthocyanin genes. Plant J. 1998;13(4):475-488. DOI: 10.1046/j. 1365-313X.1998.00046.

10. Broun P. Transcriptional control of flavonoid biosynthesis: a 
complex network of conserved regulators involved in multiple aspects of differentiation in Arabidopsis. Curr Opin Plant Biol. 2005;8(3):272-279. DOI: 10.4161/psb.27522.

11. Quattrocchio F, Wing J, van der Woude K, Souer E, de Vetten $\mathrm{N}$, Mol J, et al. Molecular analysis of the anthocyanin2 gene of petunia and its role in the evolution of flower colour. Plant Cell. 1999;11(8):1433-1444.

12. Kobayashi S, Goto-Yamamoto N, Hirochika H. Retrotransposon-induced mutations in grape skin colour. Science 2004;304(5673):982. DOI: 10.1126/science.1095011

13. Walker AR, Lee E, Bogs J, McDavid DAJ, Thomas MR, Robinson SP. White grapes arose through the mutation of two similar and adjacent regulatory genes. Plant J. 2007;49(5):772-785. DOI: 10.1111/j.1365-313X.2006.02997.

14. Kobayashi S, Goto-Yamamoto N, Hirochika H. Association of $V v m y b A 1$ gene expression with anthocyanin production in grape (Vitis vinifera) skin-colour mutants. J Jpn Soc Hortic Sci. 2005;74(3):196-203. DOI: 10.1007/s00334-014-0512-9.

15. Paz-Ares J, Ghosal D, Wienand U, Peterson PA, Saedler H. The regulatory $c l$ locus of Zea mays encodes a protein with homology to myb proto-oncogene products and with structural similarities to transcriptional activators. EMBO J. 1987;6(12):3553.

16. Espley RV, Hellens RP, Putterill J, Stevenson DE, KuttyAmma S, Allan AC. Red colouration in apple fruit is due to the activity of the MYB transcription factor, MdMYB10. Plant J. 2007;49(3):414-27. DOI: 10.1111/j.1365-313X. 2006. 02964.

17. Takos AM, Jaffé FW, Jacob SR, Bogs J, Robinson SP, Walker AR. Light-induced expression of a $M Y B$ gene regulates anthocyanin biosynthesis in red apples. Plant Physiol. 2006;142(3):1216-1232. DOI: 10.1104/pp.106.088104

18. Peel GJ, Pang Y, Modolo LV, Dixon RA. The LAP1 MYB transcription factor orchestrates anthocyanidin biosynthesis and glycosylation in Medicago. Plant J. 2009;59(1):136-149. DOI: $10.1111 / j .1365-313 X .2009 .03885$.

19. Konczak I, Zhang W. Anthocyanins-more than nature's colours. J Biomed Biotechnol. 2004;2004(5):239-240. DOI: 10.1155/S1110724304407013.

20. Jurenka JS. Therapeutic applications of pomegranate (Punica granatum L.): a review. Alternative medicine review: Alter Med Rev. 2008;13(2):128-144. DOI: 10.1080/0972060X. 2014.960272

21. Rajan S, Mahalakshmi S, Deepa VM, Sathya K, Shajitha S, Thirunalasundari T. Antioxidant potentials of Punica granatum fruit rind extracts. Int J Pharm Pharm Sci. 2011;3(3):82-88.

22. Grotewold E. The genetics and biochemistry of floral pigments. Annu Rev Plant Biol. 2006;57:761-80. DOI: 10.1146/ annurev.arplant.57.032905.105248.

23. Holton TA, Cornish EC. Genetics and biochemistry of anthocyanin biosynthesis. The Plant Cell.1995;7(7):1071. DOI:org/ 10.1105/tpc.7.7.1071.

24. Winkel-Shirley B. Flavonoid biosynthesis. A colourful model for genetics, biochemistry, cell biology, and biotechnology. Plant Physiol. 2001;126(2):485-493. DOI:org/10.1104/pp. 126.2.485.

25. Holland D, Hatib K, Bar-Ya'akov I. Pomegranate: Botany, Horticulture, Breeding. Hort Rev. 2009;35(2):127-191. DOI:
$10.1002 / 9780470593776$

26. Gil MI, García-Viguera C, Artés F, Tomás-Barberán FA. Changes in pomegranate juice pigmentation during ripening. J Sci Food Agri. 1995;68(1):77-81. DOI: 10.1002/jsfa.2740 680113.

27. Gil MI, Tomás-Barberán FA, Hess-Pierce B, Holcroft DM, Kader AA. Antioxidant activity of pomegranate juice and its relationship with phenolic composition and processing. J Agri Food chem. 2000;48(10):4581-4589. DOI: 10.1021/ jf000 404a.

28. Tzulker R, Glazer I, Bar-Ilan I, Holland D, Aviram M, Amir R. Antioxidant activity, polyphenol content, and related compounds in different fruit juices and homogenates prepared from 29 different pomegranate accessions. J Agri Food Chem. 2007;55(23):9559-9570. DOI: 10.1021/jf071413n.

29. Ben-Simhon Z, Judeinstein S, Nadler-Hassar T, Trainin T, Bar-Ya'akov I, Borochov-Neori H, Holland D. A pomegranate (Punica granatum L.) WD40-repeat gene is a functional homologue of Arabidopsis TTG1 and is involved in the regulation of anthocyanin biosynthesis during pomegranate fruit development. Planta. 2011;234(5):865-881. DOI: 10.1007/s 00425-011-1438-4.

30. Grotewold E, Drummond BJ, Bowen B, Peterson T. The mybhomologous $P$ gene controls phlobaphene pigmentation in maize floral organs by directly activating a flavonoid biosynthetic gene subset. Cell 1994;76(3):543-553. DOI: 10.1016/ 0092-8674(94)90117.

31. Grotewold E, Sainz MB, Tagliani L, Hernandez JM, Bowen $\mathrm{B}$, Chandler VL. Identification of the residues in the Myb domain of maize $C 1$ that specify the interaction with the bHLH cofactor R. PNAS. 2000;97(25):13579-13584. DOI: 10.1073/pnas.250379897.

32. Meisel L, Fonseca B, González S, Baeza-Yates R, Cambiazo $\mathrm{V}$, Campos R, et al. A rapid and efficient method for purifying high quality total RNA from peaches (Prunus persica) for functional genomics analyses. Biol Res. 2005;38(1):83-88. DOI: $10.4067 / \mathrm{S} 0716-97602005000100010$

33. Porebski S, Bailey LG, Baum BR. Modification of a CTAB DNA extraction protocol for plants containing high polysaccharide and polyphenol components. Plant Mol Biol Rep. 1997;15(1):8-15. DOI: 10.1016/j.foodchem.2015.05.004.

34. Jin J, Zhang H, Kong L, Gao G, Luo J. PlantTFDB 3.0: a portal for the functional and evolutionary study of plant transcription factors. Nucleic Acids Res. 2014;42:D1182-1187 DOI: 10.1093/nar/gkt1016.

35. Kelley LA, Sternberg MJE. Protein structure prediction on the Web: a case study using the Phyre server. Nat protoc. 2009;4(3):363-371. DOI: 10.1038/nprot.2009.

36. Ogata K, Hojo H, Aimoto S, Nakai T, Nakamura H, Sarai A, et al. Solution structure of a DNA-binding unit of Myb: a helix-turn-helix-related motif with conserved tryptophans forming a hydrophobic core. PNAS. 1992;89(14):6428-6432.

37. Deluc L, Barrieu F, Marchive C, Lauvergeat V, Decendit A, Richard T, et al. Characterization of a grapevine R2R3-MYB transcription factor that regulates the phenylpropanoid pathway. Plant Physiol. 2006;140(2):499-511. DOI: 10.1104/pp. 105.067231

38. Gómez-Maldonado J, Avila C, Torre F, Cañas R, Canovas FM, Campbell MM. Functional interactions between a gluta- 
mine synthetase promoter and MYB proteins. Plant $J$. 2004;39(4):513-526. DOI: 10.1111/j.1365-313X.2004.02153.

39. Prouse MB, Campbell MM. The interaction between MYB proteins and their target DNA binding sites. Biochim Biophys Acta (BBA)-Gene Regulatory Mechanisms. 2012;1819(1):6777. DOI: 10.1016/j.bbagrm.2011.10.010.

40. Prouse MB, Campbell MM. Interactions between the R2R3MYB Transcription Factor, At MYB61, and Target DNA Binding Sites. PLoS One. 2013;8(5):e6513. DOI: 10.1371/ journal.pone.0065132.

41. Ogata K, Morikawa S, Nakamura H, Sekikawa A, Inoue T, Kanai H, Sarai A, Ishii S, Nishimura Y. Solution structure of a specific DNA complex of the Myb DNA-binding domain with cooperative recognition helices. Cell 1994;79(4):639648. DOI: 10.1016/0092-8674(94)90549-5.

42. Dubos C, Stracke R, Grotewold E, Weisshaar B, Martin C, Lepiniec L. MYB transcription factors in Arabidopsis. Trends Plant Sci. 2010;15(10):573-581. DOI: 10.1016/j.tplants.2010. 06.005

43. Kim SH, Lee JR, Hong ST, Yoo YK, An G, Kim SR. Molecular cloning and analysis of anthocyanin biosynthesis genes preferentially expressed in apple skin. Plant Sci. 2003;165(2):403-413.

44. Allan AC, Hellens RP, Laing WA. MYB transcription factors that colour our fruit. Trends Plant Sci. 2008;13(3):99-102. DOI: $10.1016 /$ j.tplants.2007.11.012.

45. Yamazaki M, Shibata M, Nishiyama Y, Springob K, Kitayama M, Shimada N, et al. Differential gene expression profiles of red and green forms of Perilla frutescens leading to comprehensive identification of anthocyanin biosynthetic genes. FEBS J. 2008;275(13):3494-3502. DOI: 10.1111/j.17424658.2008.06496.

46. McKeown AN, Bridgham JT, Anderson DW, Murphy MN, Ortlund EA, Thornton JW. Evolution of DNA specificity in a transcription factor family produced a new gene regulatory module. Cell 2014;159(1):58-68. DOI: 10.1016/j.cell.2014. 09.003 .

47. Wissenbach M, Überlacker B, Vogt F, Becker D, Salamini F, Rohde W. MYB genes from Hordeum vulgare: tissue-specific expression of chimeric Myb promoter/Gus genes in transgenic tobacco. Plant J. 1993;4(3):411-422. DOI: 10.1046/j. 1365-313X.1993.04030411. 